\title{
Total Laparoscopic Resection by Retroperitoneal Approach Using Virtual Navigation: Two Case Reports of Primary Retroperitoneal Schwannoma
}

Yuta Sato

Gifu University

Nobuhisa matsuhashi ( $\square$ nobuhisa517@hotmail.com )

Gifu university

Yuto Sugie

Gifu University

Masashi Kuno

Gifu University

Shigeru Kiyama

Gifu Univesity

Yoshihiro Tanaka

Gifu University

Naoki Okumura

Gifu University

Takao Takahashi

Gifu Univesity

Takuya Saiki

Gifu University

Kazuhiro Yoshida

Gifu University

\section{Case report}

Keywords: Retroperitoneal schwannoma, Retroperitoneal approach, Laparoscopic resection, Virtual navigation, Navigation surgery

Posted Date: November 1st, 2021

DOI: https://doi.org/10.21203/rs.3.rs-1020263/v1

License: (c) (1) This work is licensed under a Creative Commons Attribution 4.0 International License.

Read Full License 


\section{Abstract}

Background: We report two rare cases of retroperitoneal schwannoma completely resected by laparoscopic retroperitoneal approach aided by virtual navigation.

Case presentation: These two case reports are of a 60-year-old man and a 40-year-old man with asymptomatic retroperitoneal schwannoma. In both cases, the tumors were located in the right renal hilum and were close to the duodenum, right ureter, and inferior vena cava. Simulation using threedimensional images was performed before surgery, and a retroperitoneal approach was performed to secure a wide surgical field. During the operation, we confirmed the location of the main feeder and the relationship between the tumor and organs with those shown on the three-dimensional images and performed total laparoscopic resection.

Conclusion: The retroperitoneal approach provides operative safety. Preoperative simulation and intraoperative navigation with three-dimensional images are useful methods to enhance the surgeon's understanding of a patient's specific anatomy and are especially effective when resecting a retroperitoneal tumor that is located in an anatomically deep and complex location.

\section{Background}

Schwannomas are mostly benign tumors arising from Schwann cells of the peripheral and cranial nerves and are rarely situated in the retroperitoneal space [1]. We report two rare cases of asymptomatic retroperitoneal schwannoma that were found during regular medical checkups. In both cases, the tumors were located in the right renal hilum and were surrounded by the duodenum, right ureter, and inferior vena cava (IVC). We performed a simulation using a high-speed three-dimensional (3D) image analysis system (SYNAPSE VINCENT, Fuji Photo Film Co., Ltd., Tokyo, Japan) to obtain more accurate localization of the anatomy of the tumors. Total laparoscopic resection via a retroperitoneal approach was safely performed on these two patients. Preoperative simulation with virtual navigation is a useful method to enhance the surgeon's understanding of a patient's specific anatomy and is especially effective when resecting a retroperitoneal tumor in a deep, anatomically complex location.

\section{Case Presentation}

\section{Case 1}

A 60-year-old man had a right retroperitoneal abdominal lesion that was found incidentally on abdominal computed tomography (CT) during a medical health checkup and was referred to our hospital for additional evaluation. Physical findings revealed a flat abdomen that was soft, not tender, and with no palpable mass. CT revealed a well-defined round $27 \times 24 \mathrm{~mm}$ cystic mass in the right retroperitoneum (Fig. 1a). The mass was located in the right renal hilum, dorsal to the IVC, and ventral to the right iliopsoas muscle. T2-weighted magnetic resonance imaging (MRI) showed a high-intensity lesion, with 
the main feeder being a branch of the right third lumbar artery (Fig. 1b). A mass showing low fluorodeoxyglucose (FDG) uptake (maximum standardized uptake value [SUV]: 3.74) was observed on positron emission tomography and computed tomography (PET-CT) in the same location (Fig. 1c). A tentative preoperative diagnosis of malignant tumor of undermined origin, schwannoma, or paraganglioma was considered based on these radical findings. We used the SYNAPSE VINCENT to convert Digital Imaging and Communication in Medicine (DICOM) data of the contrast-enhanced CT images to 3D images, and the locations of the lesion and main feeder were clearly identified before surgery (Fig. 1d). We performed laparoscopic resection through four ports after inducing pneumoperitoneum (Fig. 2a) and a retroperitoneal approach to provide a favorable surgical field (Fig. 2b). After incision of the retroperitoneum, the tumor was observed in the right iliopsoas muscle (Fig. 2c). After taping the right ureter, the right iliopsoas muscle was split to expose the tumor, and the feeding artery from the lumbar artery was visually recognized. We used virtual navigation to intraoperatively locate the feeding artery that led to the dorsal side of the tumor (Fig. 2d). The tumor specimen was removed via the umbilical wound (Fig. 3). The operative time was 116 minutes, and the operative blood loss was approximately $3 \mathrm{~mL}$. A pathological examination of the tumor confirmed the diagnosis of ancient schwannoma with clear surgical margins. The patient recovered without complications and was discharged on the 6th day after surgery.

\section{Case 2}

In a 40-year-old man with rheumatoid arthritis, a retroperitoneal lesion was incidentally found on a screening CT. His general physical and abdominal examinations revealed normal findings. CT revealed a solid tumor of $18 \times 17 \mathrm{~mm}$ on the dorsal side of the IVC and the right renal vein (Fig. 4a). T2-weighted MRI showed a high-intensity lesion (Fig. 4b), and a mass with low FDG uptake (max SUV: 2.54) was observed on PET-CT (Fig. 4c). Before surgery, we discussed the patient's 3D images with a urologist and performed laparoscopic surgery after fully understanding the location of the tumor (Fig. 4d). We performed a retroperitoneal approach through six ports (Fig. 5a, b). An incision in the retroperitoneum and taping of the right ureter revealed the tumor, which was in contact with the dorsal side of the IVC, but when the 3D images was rechecked intraoperatively, there was no invasion and the dissection could be performed safely (Fig. 5c). During the surgery, this 3D images was shown on a separate monitor along with the laparoscopic image, which could be freely rotated and viewed from any angles (Fig. $5 \mathrm{~d}$ ). The use of intraoperative virtual navigation was effective in identifying the tumor deep within the retroperitoneum. The cut surface of the tumor showed red-brownish cyst formation and a yellowish-white rim (Fig. 6). The operative time was 147 minutes, and the operative blood loss was approximately $5 \mathrm{~mL}$. He was discharged on the 7th day after surgery.

Written informed consent was obtained from both patients for publication of the present case reports and the accompanying images.

\section{Discussion And Conclusions}


Retroperitoneal schwannomas are rare, accounting for just $0.5-3.0 \%$ of all schwannomas, and only $1 \%$ of all peritoneal neoplasias [2]. As these tumors are usually asymptomatic and discovered by chance or in the course of the evaluation of an unrelated health problem, they may cause a delay in their early diagnosis and treatment. The ideal treatment for schwannoma is complete resection of the tumor and capsule without injuring the adherent organs [3].

With recent advances in the field of minimally invasive surgery, several laparoscopic approaches to retroperitoneal schwannomas have been reported [4, 5]. Laparoscopic surgery, which has become a useful and feasible option for this procedure, is associated with minimal invasiveness and early postoperative recovery. The retroperitoneal approach is the surgical technique for laparoscopic right colectomy [6]. The anatomical landmarks of the retroperitoneal approach are the third portion of the duodenum, the mesenteric root of the terminal ileum, and the caudal portion of the cecum. This approach begins with an incision of the peritoneum at the base of the mesentery of the intestine. After lifting the cecum or the terminal ileum ventrally, the dissection is performed just dorsal to the right fusion fascia of Told and sufficiently to the cranial side and then continues medially to the second portion of the duodenum (Fig. 7a). We decided to perform this approach based on simulating the surgery using the 3D images obtained preoperatively. The retroperitoneal approach is the preferred procedure in laparoscopic resection of retroperitoneal tumors because it involves wide dissection of the ventral side of the deep subperitoneal fascia and the surface of the duodenum and thus provides a favorable surgical field (Fig. 7b).

A retroperitoneal tumor can be in close contact with structures such as the duodenum, right renal vein, and IVC, which often require meticulous dissection to avoid damage. Thus, precise knowledge of the surgical anatomy of each patient is absolutely mandatory to ensure a safe operation. In the fields of hepatic and thoracic surgery in particular, preoperative simulation has been widely used [7-10], and the safety and efficacy of 3D images for preoperative assessment have already been described [11-13]. Preoperative simulation and intraoperative navigation with 3D images are useful methods to enhance the surgeon's understanding of a patient's specific anatomy and are especially effective when resecting a retroperitoneal tumor in an anatomically deep and complex location. Three-dimensional images can be rotated freely and visualized interactively from any angle to provide an overview of the 3D relationships of the retroperitoneal organs. In addition, information about the location of the tumor can be shared between each operator and doctors from other departments, ensuring secure resection in a narrow retroperitoneal space near important densely packed organs. This imaging technique is not only helpful for laparoscopic retroperitoneal procedures but is an excellent tool for the education of surgical trainees and for medical students studying surgical anatomy.

\section{Abbreviations}

CT

computed tomography

DICOM 
Digital Imaging and Communication in Medicine

FDG

fluorodeoxyglucose

IVC

inferior vena cava

MRI

magnetic resonance imaging

PET-CT

positron emission tomography and computed tomography

SUV

standardized uptake value

3D

three-dimensional.

\section{Declarations}

\section{Ethics approval and consent to participate}

These two case reports were approved by the ethics committee of Gifu University School of Medicine.

\section{Consent for publication}

Written informed consent was obtained from the patients for publication of these case reports and accompanying images. A copy of the patient's written consent is available for review by the Editor-in Chief of this journal.

\section{Availability of data and materials}

Not applicable

\section{Competing interests}

All authors declare that they have no conflict of interest.

\section{Funding}

Funding information is not applicable.

\section{Authors' contributions}

YSa drafted the manuscript. NM and YSu performed the operation. MK and SK participated in the operation. YT, NO, TT, TS and KY provided academic advice. All authors read and approved the final manuscript. 


\section{Acknowledgements}

We thank Rise Japan LLC for the English language editing.

\section{References}

1. Das Gupta TK, Brasfield RD, Strong EW, et al. Benign solitary schwannomas (neurilemomas). Cancer. 1969;24:355-66.

2. Mastoraki A, Toska F, Tsiverdis I, et al. Retroperitoneal schwannomas: dilemmas in diagnostic approach and therapeutic management. J Gastrointest Cancer. 2013;44:371-4.

3. Li Q, Gao C, Juzi JT, et al. Analysis of 82 cases of retroperitoneal schwannoma. ANZ J Surg. 2007;77:237-40.

4. Maruyama T, Sugamoto Y, Miyagishima D, et al. Laparoscopic resection of a retroperitoneal schwannoma located in the hepatic hilus. Surg Case Rep. 2015;1:18.

5. Funamizu N, Sasaki A, Matsumoto T, et al. Laparoscopic resection of a retroperitoneal schwannoma behind the lesser omental sac. Surg Laparosc Endosc Percutan Tech. 2004;14:175-7.

6. Mike M, Kano N. Laparoscopic surgery for colon cancer: a review of the fascial composition of the abdominal cavity. Surg Today. 2015;45:129-39.

7. Yamanaka J, Saito S, limuro Y, et al. The impact of 3-D virtual hepatectomy simulation in living-donor liver transplantation. J Hepatobiliary Pancreat Surg. 2006;13:363-9.

8. Mochizuki K, Takatsuki M, Soyama A, et al. The usefulness of a high-speed 3D-image analysis system in pediatric living donor liver transplantation. Ann Transplant. 2012;17:31-4.

9. Chen-Yoshikawa TF, Date H. Update on three-dimensional image reconstruction for preoperative simulation in thoracic surgery. J Thorac Dis. 2016;8:295-301.

10. Ikeda N, Yoshimura A, Hagiwara $\mathrm{M}$, et al. Three dimensional computed tomography lung modeling is useful in simulation and navigation of lung cancer surgery. Ann Thorac Cardiovasc Surg. 2013;19:15.

11. Lawler LP, Fishman EK. Multi-detector row CT of thoracic disease with emphasis on 3D volume rendering and CT angiography. Radiographics. 2001;21:1257-73.

12. Yao F, Wang J, Yao J, et al. Three-dimensional image reconstruction with free open-source OsiriX software in video-assisted thoracoscopic lobectomy and segmentectomy. Int J Surg. 2017;39:16-22.

13. Nia PS, Olsthoorn JR, Heuts S, et al. Interactive 3D reconstruction of pulmonary anatomy for preoperative planning, virtual simulation, and intraoperative guiding in video-assisted thoracoscopic lung surgery. Innovations, 2019;14:17-26.

\section{Figures}



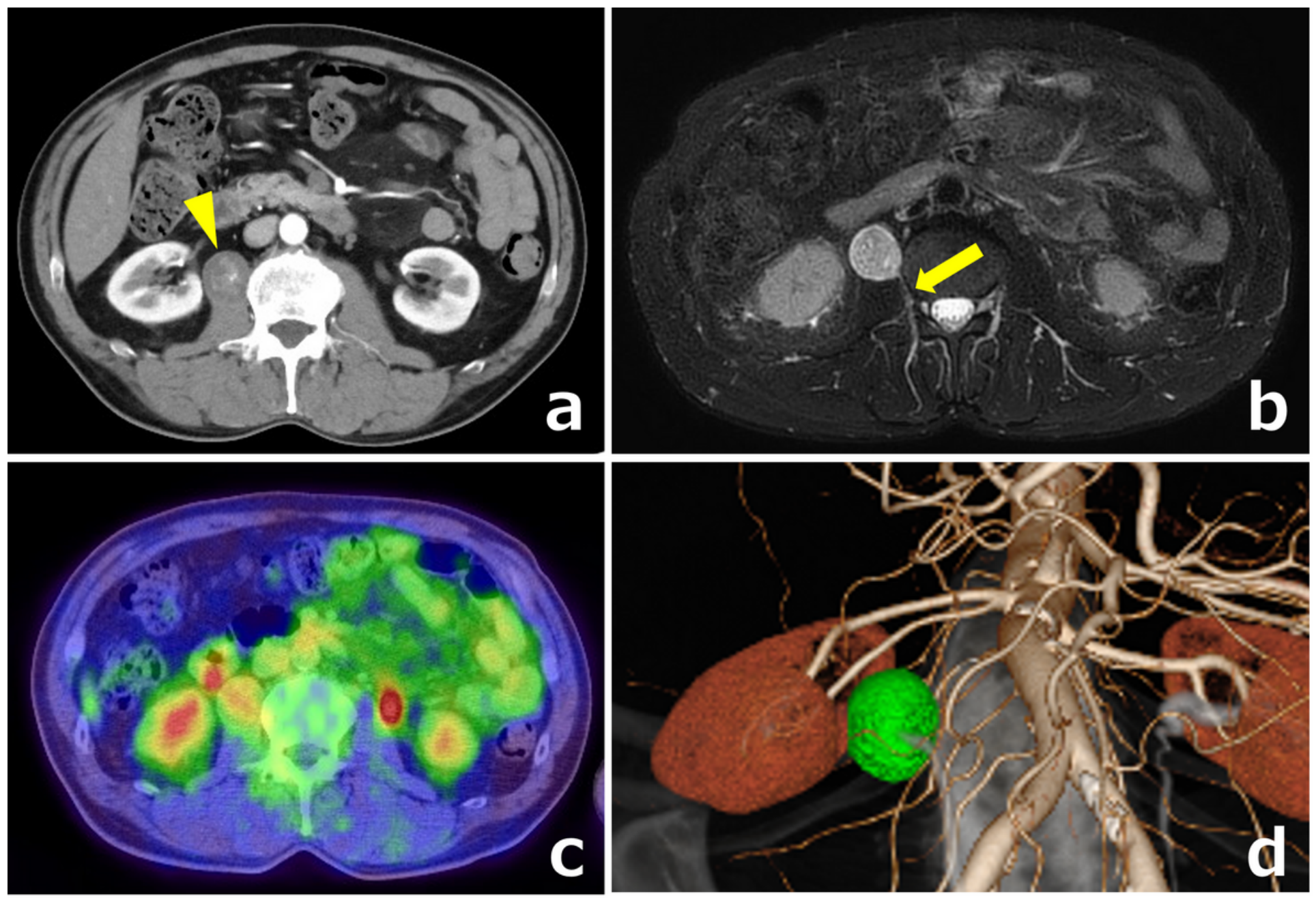

Figure 1

a CT showed a well-defined round $27 \times 24 \mathrm{~mm}$ cystic mass (yellow arrowhead). b T2-weighted MRI showed a high-intensity lesion with the main feeder being a branch of the right third lumbar artery (yellow arrow). c PET-CT showed a mass with low FDG uptake. $d$ The location of the tumor and main feeder were identified before surgery on 3D images. 


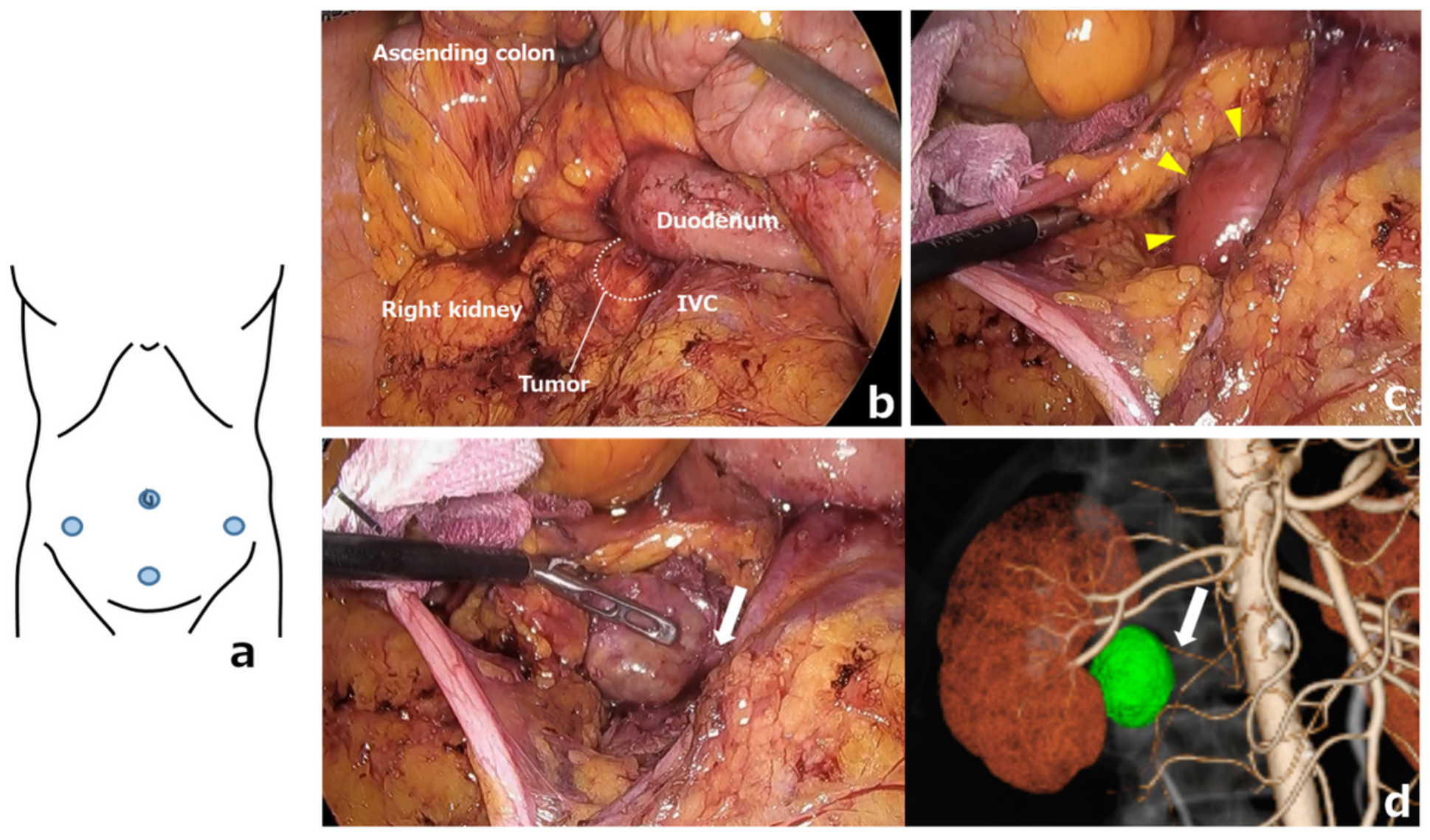

Figure 2

a Placement of the four ports. b Laparoscopic view via the retroperitoneal approach. $c$ The tumor was in the right iliopsoas muscle (yellow arrowheads). $d$ Comparison of the main feeder shown by 3D imaging and as seen in the operative view. The feeding artery (white arrow) depicted on the 3D images was consistent with that seen in the actual operative field.

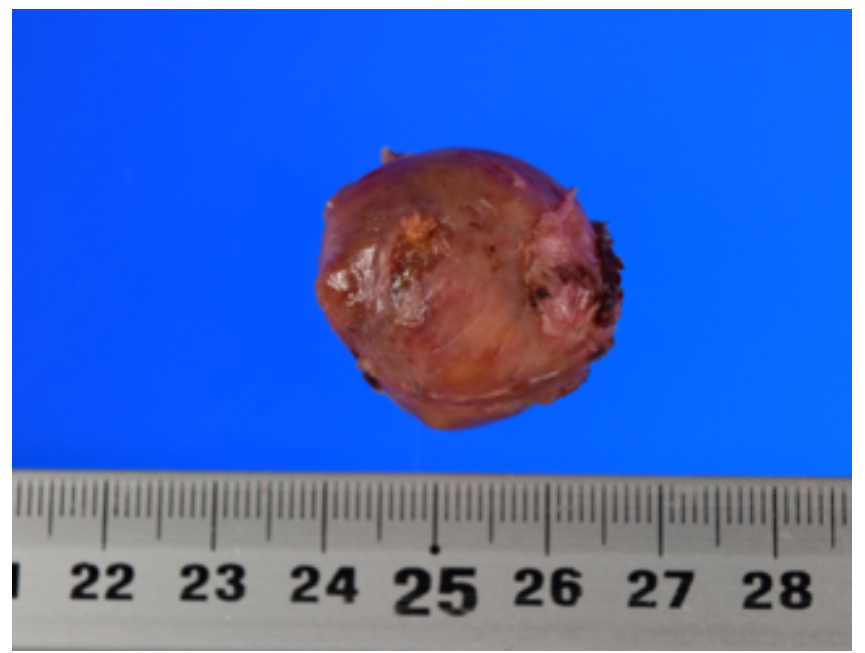

Figure 3

Surgical specimen. 

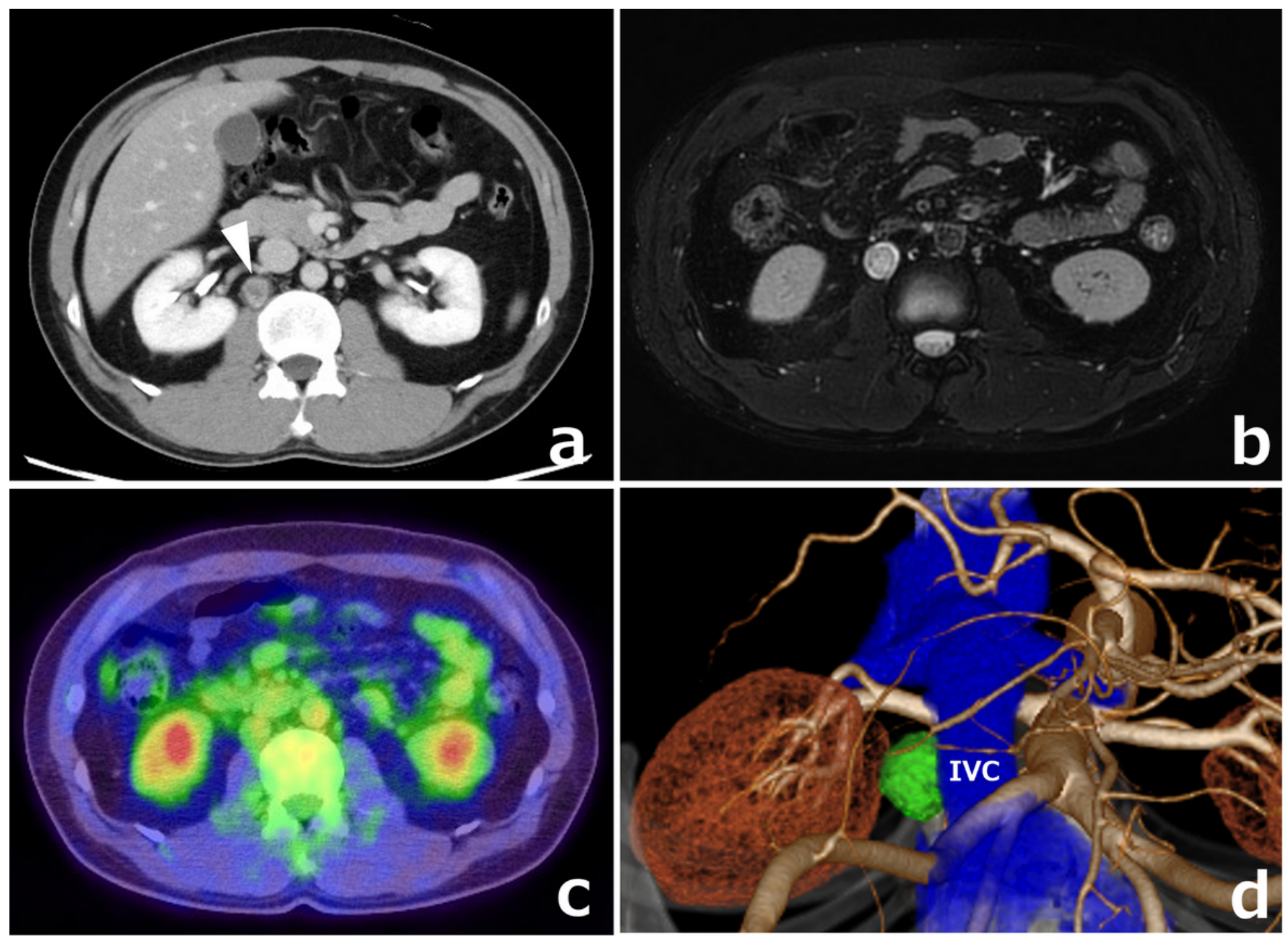

Figure 4

a CT showed a tumor (white arrowhead) on the dorsal side of the IVC. b T2-weighted MRI showed a highintensity lesion. c A mass with low FDG uptake was observed on PET-CT. d SYNAPSE VINCENT was used to convert DICOM data of the CT images to 3D images. 

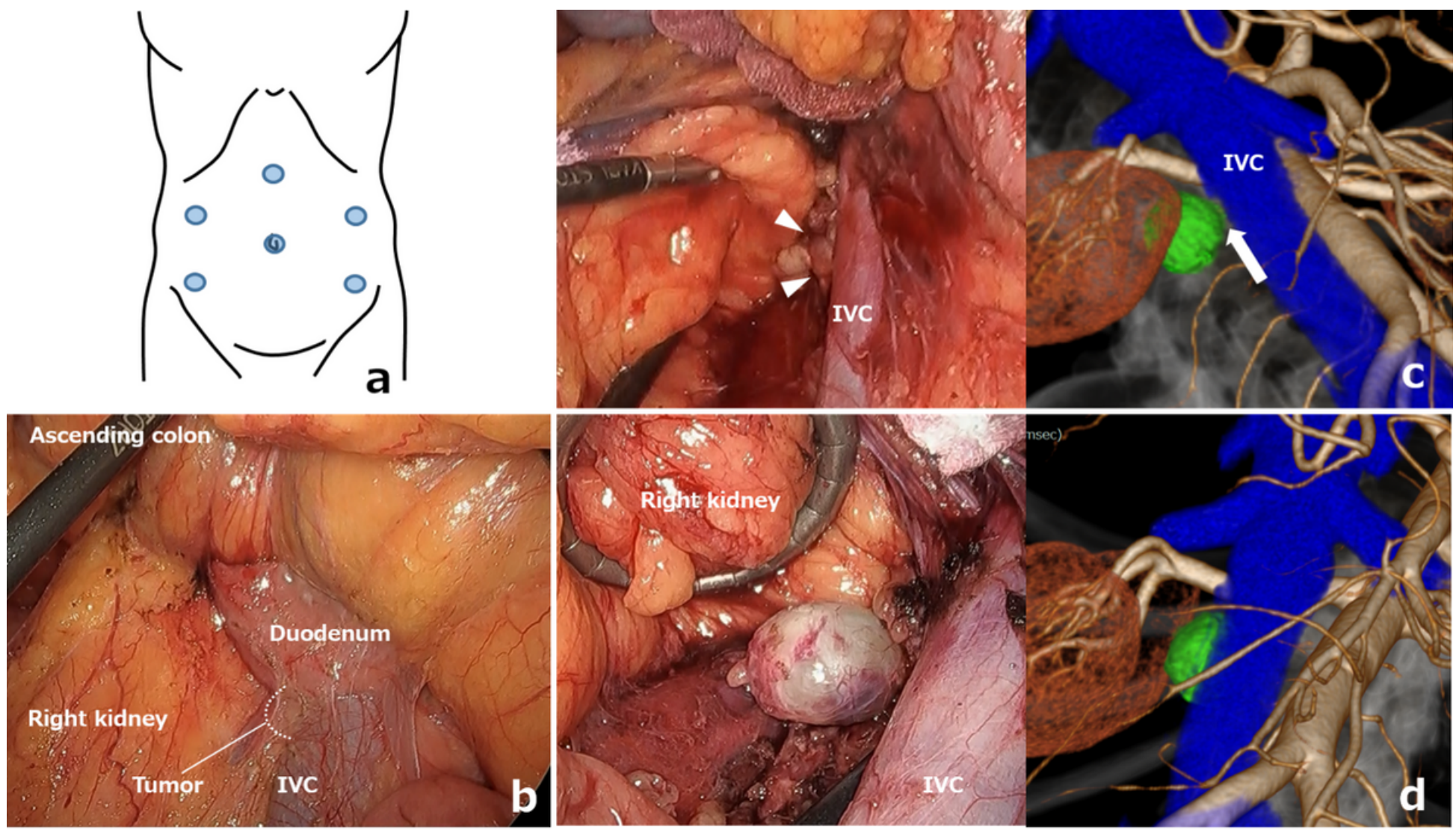

\section{Figure 5}

a Placement of the six ports. b Laparoscopic view via the retroperitoneal approach. c Comparison of the tumor shown by 3D imaging and as seen in the operative view. The tumor was on the dorsal side of the IVC (white arrowheads), but no invasion was shown on the 3D image (white arrow). $d$ Comparison of the $3 \mathrm{D}$ image and operative view.

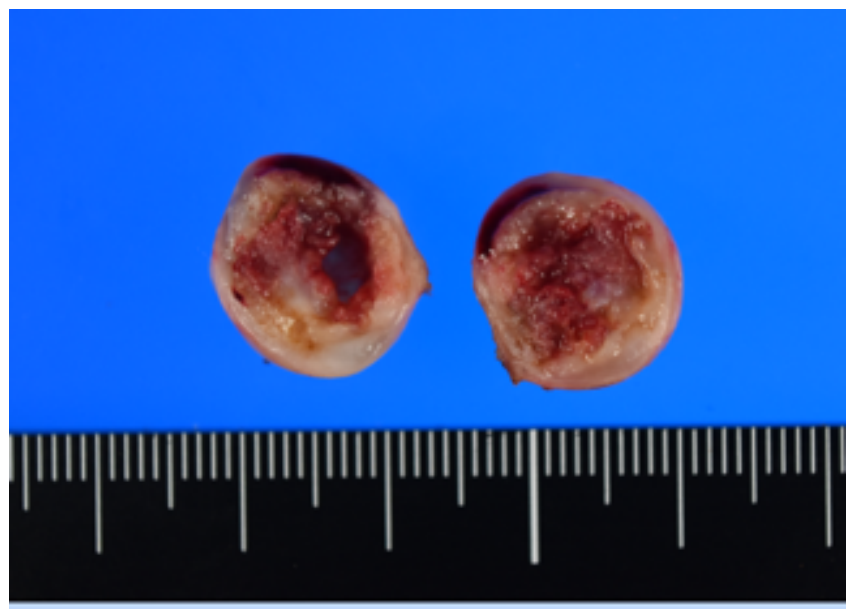

Figure 6

Surgical specimens. 

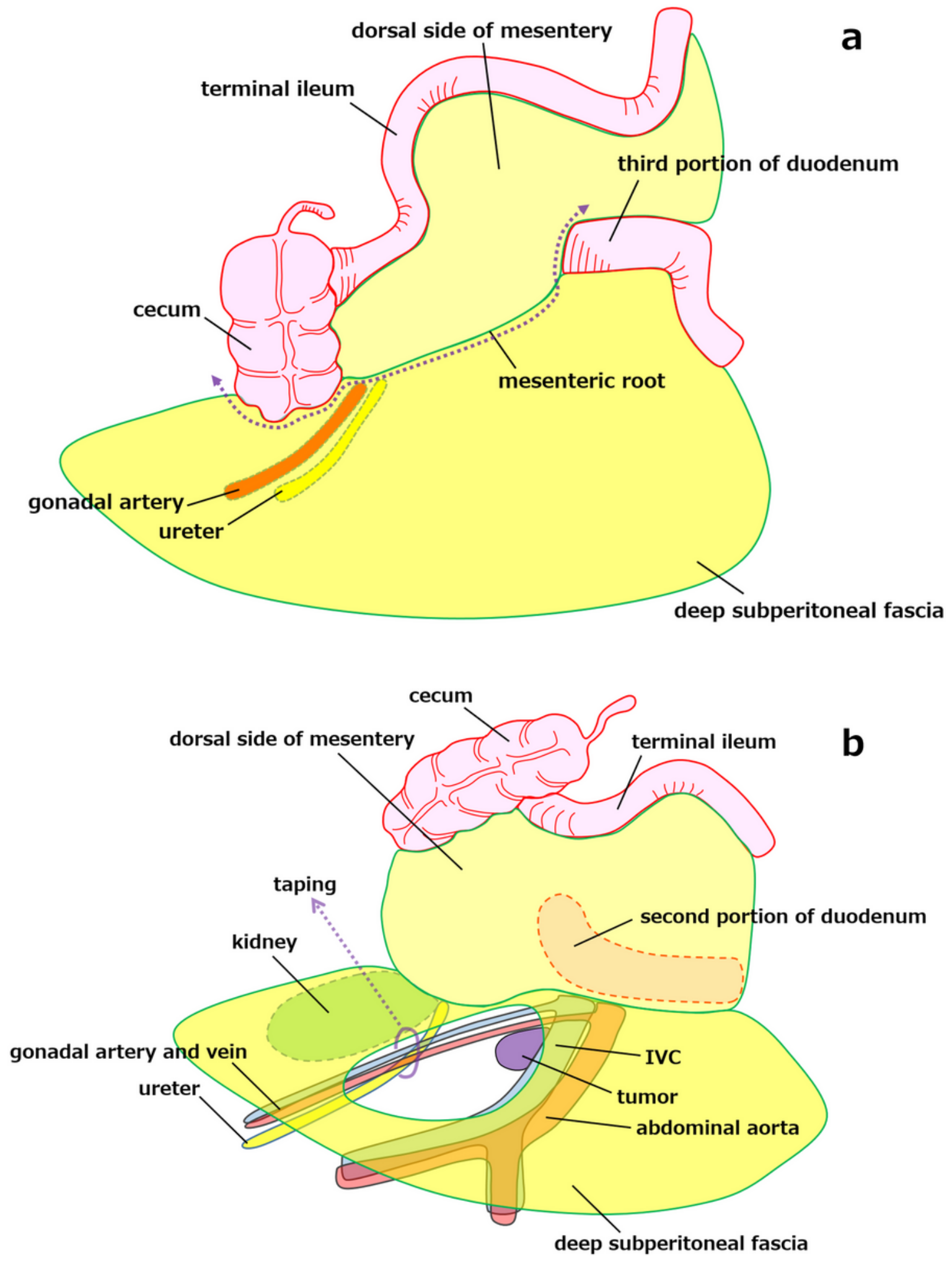

\section{Figure 7}

a The retroperitoneal approach begins with an incision of the peritoneum at the mesenteric root (dotted arrow), after which the dissection plane can be entered to the ventral side of the deep subperitoneal fascia. $b$ This approach involves wide dissection of the ventral side of the deep subperitoneal fascia and provides a favorable surgical field. 